\title{
DETECCIÓN AUTOMÁTICA DE FALLOS MEDIANTE MONITORIZACIÓN Y OPTIMIZACIÓN DE LAS FECHAS DE LIMPIEZA PARA INSTALACIONES FOTOVOLTAICAS
}

\author{
J. Segarra-Tamarit, E. Pérez, H. Beltrán, E. Belenguer ${ }^{1}$ y J.Gandía ${ }^{2}$ \\ ${ }^{1}$ DESID, Universitat Jaume I, Castelló de la Plana, España, pereze@uji.es \\ ${ }^{2}$ Greenpower Partners
}

\section{Resumen}

Este trabajo presenta una propuesta para mejorar la automatización del mantenimiento de plantas fotovoltaicas. Esto se lleva a cabo, por una parte, seleccionando los índices adecuados para la detección de fallos utilizando los datos de monitorización disponibles. Por otra parte, se estudia el efecto de la suciedad en los paneles y se plantea un método para optimizar las fechas y frecuencia de limpieza de las instalaciones.

Palabras clave: Fotovoltaica, detección de fallos, optimización, limpieza de instalaciones.

\section{INTRODUCCIÓN}

En los últimos años, las energías renovables se han afianzado a nivel mundial. En 2016 se instalaron 161 GW, superándose los valores de años anteriores y alcanzando un total de $2006 \mathrm{GW}$ [1].

Aunque hay un estancamiento de la construcción de nuevos proyectos en Europa, a nivel mundial la energía fotovoltaica ha seguido creciendo gracias en gran medida a China, Japón y a países en desarrollo [2]. Debido a que se realizan menos instalaciones nuevas, el sector en Europa se está especializando en ingeniería, estudios técnicos, administración de activos y operación y mantenimiento [3].

Entre las ventajas de la energía fotovoltaica se encuentra que, gracias a su carácter modular, se pueden construir instalaciones en prácticamente cualquier lugar y de una gran variedad de potencias. Esto permite que existan desde pequeñas instalaciones para el autoconsumo, o integradas arquitectónicamente, hasta centrales de gran potencia. Además, su vida útil es superior a los 20 años y, con un buen mantenimiento, se puede prolongar hasta los 40 años en algunos casos [4].

En este trabajo se pretende mejorar dos aspectos fundamentales del mantenimiento de instalaciones fotovoltaicas como son la detección automática de fallos mediante los datos de monitorización y la optimización de las fechas de limpieza. Para ello, en la sección 2 se describen los componentes principales de una instalación, su funcionamiento y los fallos asociados a cada uno de ellos. En la sección 3 , se analizan los dos índices utilizados para la detección de fallos, así como algunas de sus limitaciones debidas a la variabilidad o la presencia de sombras sobre algunos paneles. En la sección 4 se define una función de coste para minimizar las pérdidas debidas al efecto de la suciedad. Finalmente, en las secciones 5 y 6 se muestran resultados obtenidos tanto en la detección de fallos en instalaciones reales como en la optimización, y se ofrecen algunas conclusiones.

\section{DESCRIPCIÓN DEL SISTEMA Y PLANTEAMIENTO DEL PROBLEMA}

Las instalaciones fotovoltaicas están compuestas por paneles solares, que se conectan en serie para aumentar la tensión hasta los valores de entrada del inversor. Las series de paneles pueden estar montadas directamente sobre una cubierta o sobre estructuras de soporte que les dan la inclinación deseada para captar mejor la radiación solar. Estas estructuras soportan una o varias series de paneles sobre ellas y están espaciadas entre sí para intentar evitar que se hagan sombras entre ellas.

Las series se conectan en paralelo para que la corriente también tenga valores manejables por el inversor. Esto se hace en cajas de conexiones, que contienen además protecciones para cada una de las series que llegan. Es frecuente que en centrales fotovoltaicas haya varios niveles de cajas. En un primer nivel se unen las series y en el segundo se unen las salidas de las cajas anteriores para poder conectarlas al inversor. Dependiendo de la instalación puede haber instrumentos de monitorización instalados en las cajas que permitan conocer la corriente de cada serie en tiempo real. Con este nivel de monitorización es sencillo detectar el fallo de una serie, ya que solamente hay que comprobar si la serie está generando corriente o no.

Por último, se encuentran los inversores. Estos transforman la corriente continua en alterna y ge- 
neralmente tienen monitorizadas todas sus variables de funcionamiento. Además, si se trata de una central conectada a la red que requiere aumentar la tensión, también se requiere un centro de transformación.

En una central fotovoltaica se pueden producir fallos de varios tipos. Uno relativamente común que hay que controlar es que no se desconecten o rompan los terminales que unen los paneles y que las protecciones de las cajas (normalmente fusibles) no se hayan desconectado. Estos fallos son difíciles de detectar a través de la monitorización a nivel de inversor porque la instalación sigue funcionando pese al defecto, aunque produciendo una potencia menor.

Otro de los posibles puntos de fallo son los inversores. Cuando falla uno, se pierde la producción de toda la parte de la instalación que está conectada a él. Este tipo de fallo es más fácil de detectar, ya que supone un cambio grande en la evolución de la potencia inyectada por la planta.

Otro aspecto importante en relación al mantenimiento de las instalaciones fotovoltaicas es que, como los paneles están en el exterior, se van ensuciando con el paso del tiempo y para evitar la pérdida de producción que ello implica es habitual que se limpien los paneles al menos una vez al año. Esta limpieza es conveniente hacerla en el momento óptimo del año para obtener un mayor impacto en los beneficios.

\section{DETECCIÓN AUTOMÁTICA DE FALLOS MEDIANTE ÍNDICES DE RENDIMIENTO}

\subsection{Performance Ratio}

Uno de los índices más utilizados para comprobar el funcionamiento de instalaciones fotovoltaicas es el Performance Ratio $(P R)$, que compara la energía producida por la instalación $(E)$ en un periodo determinado con la que podría producir de manera teórica $\left(E_{t e o}\right)$ en ese mismo periodo.

$$
P R=\frac{E}{E_{\text {teo }}}
$$

Para calcular $\left(E_{t e o}\right)$, el primer paso es obtener la potencia teórica $\left(P_{\text {teo }}\right)$ en cada instante de tiempo, utilizando la irradiancia medida por los piranómetros de la instalación.

$$
P_{\text {teo }}(t)=\frac{\text { Irradiancia }(t)}{1000} P_{p}
$$

Dónde $P_{p}$ es la potencia pico de la instalación. La energía teórica se obtiene integrando la potencia durante el periodo deseado $(T)$.

$$
E_{\text {teo }}=\int_{0}^{T} P_{t e o}(t) d t
$$

El $P R$ se puede calcular diariamente para comprobar el rendimiento de la instalación. Sin embargo, este índice presenta variaciones a lo largo del año de hasta un $20 \%$ debido, entre otros factores, a la suciedad acumulada en los paneles o las pérdidas de rendimiento por temperatura [5]. Además, en un mismo mes también puede variar cerca de un $10 \%$ de unos días a otros.

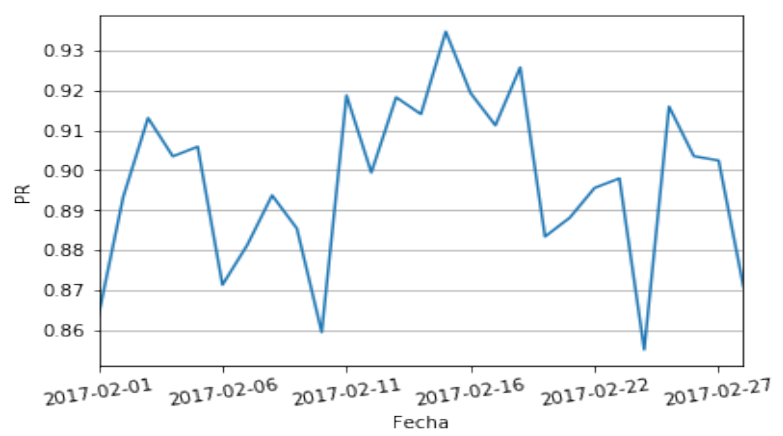

Figura 1: Variaciones del $P R$ durante un mes

Pese a estas variaciones, el $P R$ se suele calcular diariamente para detectar fallos importantes como que se desconecte un inversor. Por ejemplo, la Figura 1 muestra la evolución del $P R$ a lo largo de un mes de funcionamiento normal. Se puede entender que, a pesar de las variaciones habituales, si el $P R$ bajara de 0.8 estaría relacionado con algún defecto en la instalación.

La ventaja del $P R$ es que se puede calcular midiendo únicamente la energía producida y la irradiancia.

\subsection{Ratio específico}

Para poder detectar fallos más concretos, como la rotura de un conector o de una protección que desconecte una serie de paneles o una caja, se divide la instalación en partes de las que se puedan obtener medidas. Esto va desde hacer un grupo por cada inversor de la instalación, hasta dividirla por cajas, dependiendo de las características de cada una.

Es frecuente que los diferentes grupos no tengan la misma potencia, por lo que para poder compararlos se calcula el ratio específico $(R E)$, que se mide en horas de producción u horas solares pico 
$(H S P)$. Esto consiste en dividir la energía producida por cada grupo en el periodo para el que se va a hacer la comparación, por su potencia pico.

$$
R E_{\text {grupo }}=\frac{E_{\text {grupo }}}{P_{p_{\text {grupo }}}}
$$

Una vez se conoce el valor de $R E$ de cada grupo, para compararlos de manera mas sencilla, se dividen todos por el mayor de ellos, que es la zona de la instalación que mejor está funcionando.

$$
R_{\text {grupo }}=\frac{R E_{\text {grupo }}}{R E_{\max }}
$$

Así, se obtienen valores de ratio $(R)$ entre cero y uno que representan el rendimiento de los diferentes grupos. Estos valores se pueden relacionar con la potencia de las partes que componen cada grupo. Por ejemplo, si uno tiene un ratio de $0.94 \mathrm{y}$ está formado por 17 series (una serie es el 5.8\% de la potencia total), se puede determinar que está fallando una serie de esa parte del parque.

En este cálculo se está asumiendo que la zona que tiene el ratio específico más alto no tiene ningún defecto. Esto no supone un problema grave, ya que en el momento en el que se reparase algún fallo en cualquier otra parte, la parte reparada pasaría a ser la de mayor $R$ y se detectaría el fallo en la zona que antes se suponía en buen estado.

\subsection{Sombras}

Es posible que algún elemento de los alrededores de la instalación, como un árbol o un muro de los alrededores del recinto, provoque sombras en algunas zonas. Este efecto es más frecuente en los meses de invierno, ya que el sol tiene menos elevación. Si las sombras afectan a una parte de la instalación y a otras no, o no afectan por igual, se podría detectar falsos fallos utilizando los índices anteriores. Por esto es importante identificar la presencia de sombras y descartar del cálculo de los indicadores de rendimiento las horas durante las que afectan dichas sombras.

En la Figura 2 se representa la intensidad producida por tres series de paneles de una instalación. Se puede apreciar que la serie afectada por sombras de la estructura de soporte que tiene delante produce corrientes más bajas durante las primeras y últimas horas del día, cuando el sol está más bajo. En cambio, el resto del día funciona correctamente. Por otra parte, se representa una serie afectada por un bosque cercano. Por la mañana las sombras son mayores, por lo que la producción es muy baja, mientras que el resto del día produce más corriente pero sigue afectada.

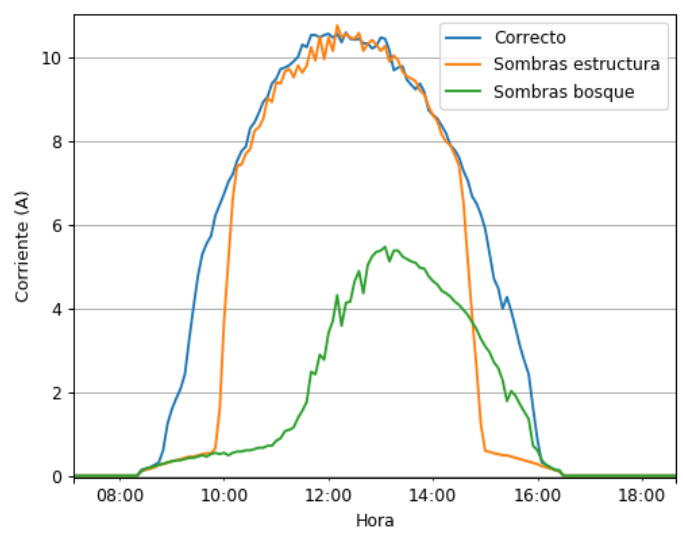

Figura 2: Corrientes de series con sombras

El efecto de las sombras también es visible a nivel de inversor. En la Figura 3 se muestra como el inversor 1 tiene menos producción entre las 8:00 y las 10:30 debido a las sombras.

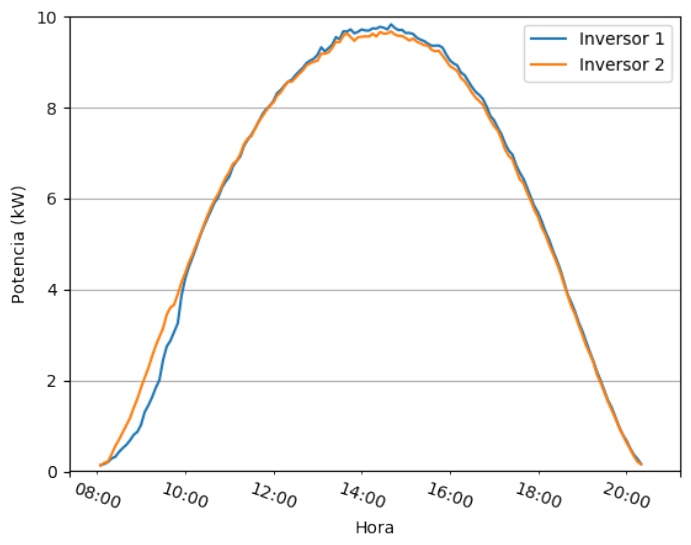

Figura 3: Potencia de inversor con sombras

Como la altura del sol es menor durante los meses de invierno, en ocasiones las sombras solo afectan durante esa época del año y desaparecen en los meses más próximos al verano. También es posible que el efecto de las sombras persista durante todo el año. En ambos casos es interesante detectar en qué época del año empieza a suceder el fenómeno o si afectan durante la mayor parte del día a lo largo del invierno, para poder descartar las horas de sombras de los cálculos o tener en cuenta a qué porcentaje de la zona de estudio afectan para descontarlo de la potencia total. 


\section{OPTIMIZACIÓN DE LAS LIMPIEZAS}

El primer paso para averiguar cuándo es conveniente limpiar una instalación es definir una función que describa cómo se reduce el rendimiento de los paneles con el paso del tiempo. Para ello, se define $L(d)$ como las pérdidas de rendimiento de los paneles por cada día que pasa desde la última limpieza.

$$
L(d)=K \cdot d
$$

Dónde $K$ son las pérdidas de rendimiento diarias. En [6] y [7] se estudia este efecto de la suciedad sobre el rendimiento, obteniéndose unos valores de $K$ de alrededor de un $0.26 \%$, que es el valor que se va a utilizar.

Por otra parte, para realizar un análisis más completo, debe considerarse que las lluvias tienen un efecto limpiador en los paneles. Por ello, en verano se alcanzan las mayores pérdidas, al encadenarse más días sin llover.

Para introducir este factor en el estudio, se define la probabilidad de que llueva en un día determinado como $q(t) \mathrm{y}$, por tanto, la de que no llueva como $p(t)=1-q(t)$. A continuación, para un día cualquiera en el que se llevan $d$ días desde la última limpieza, se pueden definir las pérdidas de rendimiento esperadas $t$ días después como:

$$
L E(t, d)=\mathbb{E}(L(t))
$$

Donde $\mathbb{E}$ representa la esperanza matemática.

Para calcular esta cantidad puede deducirse que, cuando ha pasado un día, el valor de $L$ será 0 , si ese día llueve, o $K \cdot(d+1)$ si no. Es decir, el valor esperado será:

$$
\begin{aligned}
L E(1, d) & =q(1) \cdot 0+p(1) \cdot(K \cdot(d+1))= \\
& =p(1) \cdot(K \cdot(d+1))
\end{aligned}
$$

Análogamente, para dos días el valor de $L$ será 0 si llueve ese mismo día, $K \cdot 1$ si llueve el día anterior o $K \cdot(d+2)$ si no llueve ninguno de los dos días. Por tanto se tiene:

$L E(2, d)=p(2) \cdot(q(1) \cdot K+p(1) \cdot K \cdot(d+2))$

$L E(2, d)=p(2) \cdot(q(1) \cdot K+p(1) \cdot K \cdot(1+d+1))$

$L E(2, d)=p(2) \cdot(K+p(1) \cdot K \cdot(d+1))$

$L E(2, d)=p(2) \cdot(K+L E(1, d))$

Si se calcula el valor de $L E$ para más días, puede comprobarse que existe una formulación general recursiva:

$$
L E(t, d)=p(t) \cdot(K+L E(t-1, d))
$$

A partir de esta formulación recursiva puede comprobarse que, si $p(t)$ toma una valor constante $p$, $L E$ también tiende a un valor final constante $L E_{f}$ que puede calcularse como:

$$
\begin{gathered}
L E_{f}=p \cdot\left(K+L E_{f}\right) \\
L E_{f}=\frac{p \cdot K}{(1-p)}=\frac{p \cdot K}{q}
\end{gathered}
$$

Si se toma un periodo de tiempo $(T)$ para el que se desea optimizar las fechas de limpieza, se puede calcular $L E$ para cada día del intervalo de tiempo entre limpiezas. Actualizando el valor de $d$ cada vez que se limpia la instalación se obtiene un perfil de las pérdidas esperadas para cada día.

$$
P E(t, D)= \begin{cases}E(t) \cdot L E\left(t, T-D_{n}\right) & D_{1}>t \\ E(t) \cdot L E\left(t-D_{1}, 0\right) & D_{2}>t \geq D_{1} \\ E(t) \cdot L E\left(t-D_{2}, 0\right) & D_{3}>t \geq D_{2} \\ \cdot & \\ \cdot & \\ E(t) \cdot L E\left(t-D_{n}, 0\right) & t \geq D_{n}\end{cases}
$$

Dónde $\mathrm{D}$ es un vector que contiene los días del año en que se limpia la instalación $D=\left[D_{1}, D_{2} \ldots D_{n}\right]$ y $E(t)$ representa la energía producida por la planta en el día $t$.

Con las pérdidas de energía debidas a la suciedad anteriores, el problema consiste en minimizar las pérdidas económicas $(J)$, que se obtiene de multiplicar, para cada día del año, $P E$ por el coste de la energía $\left.\left(C_{E n e r g i a}\right)\right)$ que se ha considerado constante y de $64.08 € / M W h$. Esta retribución se ha obtenido a partir del precio medio de mercado y las ayudas estatales durante el año 2016.

$$
J=\sum_{t=1}^{T} C_{\text {Energia }} \cdot P E(t, D)
$$

Este cálculo se puede hacer para instalaciones de diferente potencia, variando el número de limpiezas anuales, así como los días en los que se realizan las mismas, la velocidad con la que se ensucian los paneles y la probabilidad de lluvia.

\section{RESULTADOS}

\subsection{Detección de fallos}

Calculando diariamente los índices descritos en la sección 3 ha sido posible detectar de manera automática diferentes fallos en una planta fotovoltaica real. Mediante el $P R$ es posible detectar casos en los que un inversor deja de funcionar durante 


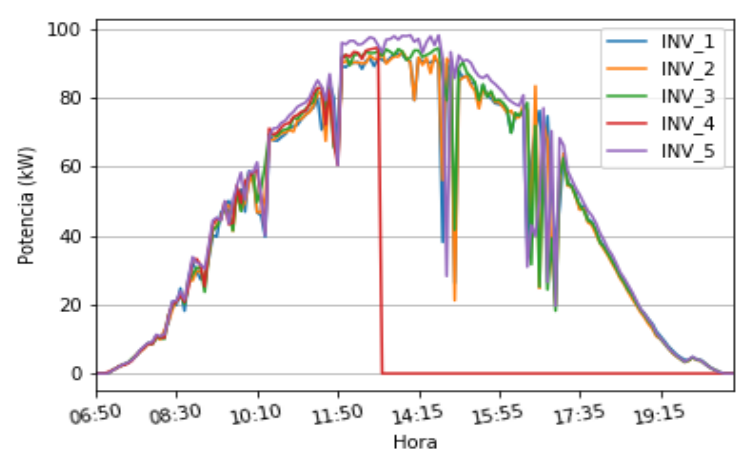

Figura 4: Fallo en un inversor

parte de un día, tal y como se muestra en la Figura 4.

En la Figura 5 se muestra como el día 16 el $P R$ bajó hasta 0.74 por el motivo descrito anteriormente y, una vez reparado el problema, volvió a los valores habituales.

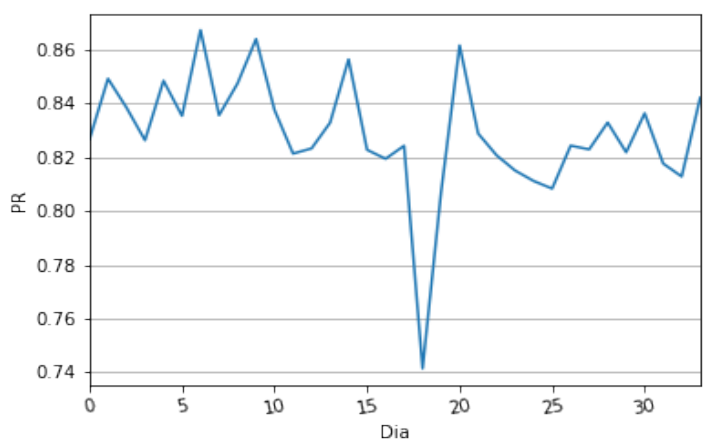

Figura 5: Variación del $P R$ al fallar un inversor

Por lo que respecta al índice $R$, en la Figura 6 se muestra como inicialmente los inversores 2,4 y 5 funcionaban entre un 4 y un $5 \%$ por debajo del inversor 3, mientras que el inversor 1 lo hacía un $10 \%$ por debajo. En el día 4 se produce la reparación de partes de la instalación, lo que mantiene algunas partes desconectadas provocando un descenso de los ratios. Durante esta reparación se sustituye 1 fusible fundido en series de los inversores 2,4 y 5 , y en el inversor 1 se cambian 2 fusibles. Esta reparación hace que en los días siguientes los inversores funcionen con ratios similares.

Otro caso de detección de fallo se muestra en la Figura 7. Al desconectarse una serie del inversor 1 , su rendimiento baja de un día a otro un $4 \%$ aproximadamente, lo que permite detectar el fallo. $\mathrm{Al}$ saber en qué zona de la instalación está el fallo se puede ahorrar tiempo en la reparación, porque se encuentra el defecto revisando únicamente las series del inversor cuyo ratio ha bajado. Durante

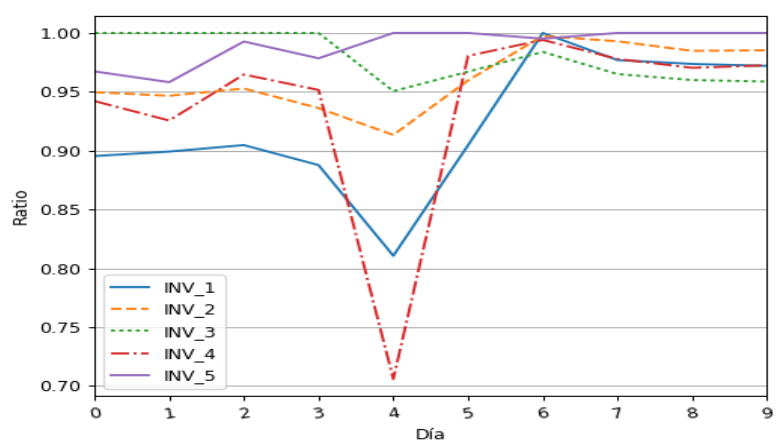

Figura 6: Variación del ratio al sustituir varios fusibles

el día 7 se realiza la reparación, por lo que el ratio ya mejora ligeramente y el día 8 ya recupera sus valores normales.

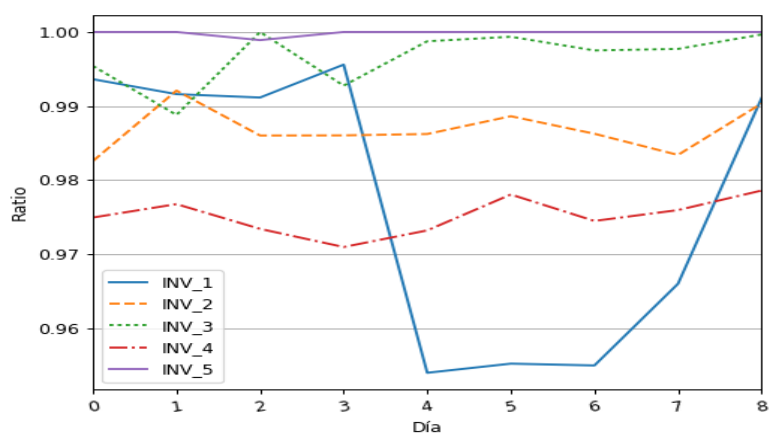

Figura 7: Variación del ratio al fundirse y cambiarse un fusible

\subsection{Optimización de las limpiezas}

Se dispone de datos de energía producida durante el año 2016 por una instalación de $583 \mathrm{kWp}$ situada en España, así como de las precipitaciones diarias de ese mismo año.

En una primera aproximación se calcula una probabilidad diaria de lluvia para todo el año del $7.92 \%$ constante, sabiendo que llovió en 29 días de los 366 .

Para comparar el efecto de la limpieza, primero se calculan las pérdidas anuales en caso de que no se limpiara nunca la instalación. Para eso se obtienen las pérdidas de rendimiento esperadas, que al ser constante la probabilidad de lluvia, tienden a un valor final constante $L E_{f}$ como se ha descrito en (12).

Así, se obtiene un valor de $L E_{f}=3.1 \%$ que, multiplicado por la energía anual y su coste, da unas pérdidas económicas de $1588.95 €$.

Limpiando los paneles una vez al año, las pérdidas varían en función del día del año en que se hace la 
limpieza tal y como se muestra en la Figura 8. El mínimo en este caso se obtiene para el día 20 de mayo con un coste de $1511.05 €$. El peor día para limpiar en este caso es el 15 de diciembre con unas pérdidas de $1575.95 €$.

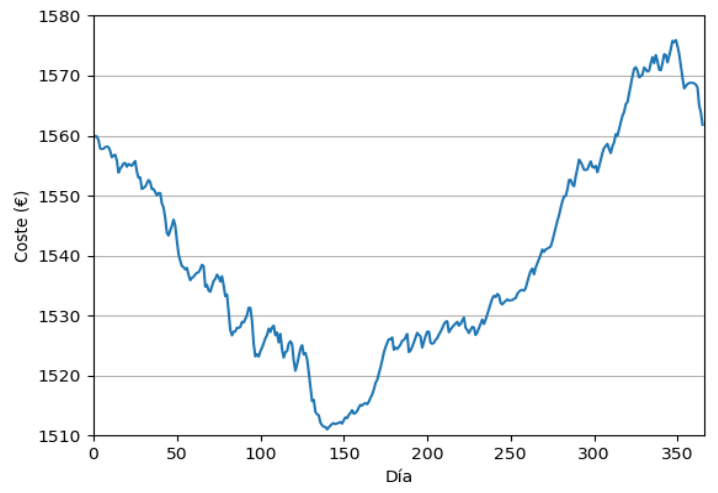

Figura 8: Coste asociado a las pérdidas por suciedad en función del día de limpieza

De los resultados obtenidos puede comprobarse que, con las probabilidades de lluvia consideradas, una limpieza de los paneles sólo resulta rentable si el coste de realizarla es muy bajo. No obstante, es habitual que los contratos de mantenimiento de plantas fotovoltaicas incluyan, entre otros servicios, una limpieza de paneles anual. En este caso, es interesante calcular el día óptimo para realizar esta limpieza de una manera más precisa.

Para ello, se han considerado probabilidades de lluvia diferentes para varios periodos del año. En concreto, a partir de análisis de históricos de lluvia en la localización de la planta, se han considerado unas probabilidades del $12 \%$ en diciembre, enero y febrero, del $2 \%$ en junio, julio y agosto y del $9 \%$ el resto del año.

En este caso no se obtiene un valor constante de $L E$ cuando no se limpia nunca la instalación, sino un perfil que varía a lo largo del año al hacerlo la probabilidad de lluvia. En la Figura 9 se observa que las pérdidas de rendimiento son mayores en los meses de verano, cuando la probabilidad de lluvia es menor, llegando a un máximo del $11.49 \%$ el día 31 de agosto. Por el contrario, las pérdidas en invierno son las más bajas. Las pérdidas económicas anuales calculadas si no se limpia son de 2389.04 $€$.

En este caso, los costes en función de en qué día del año se realiza la limpieza se muestran en la Figura 10. El mejor día para limpiar la instalación es el 16 de Julio, con unas pérdidas de 1868.26€.

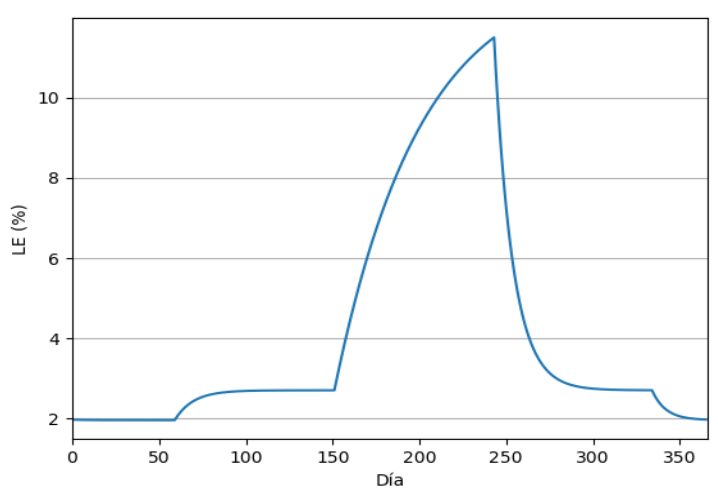

Figura 9: $L E$ con probabilidades variables

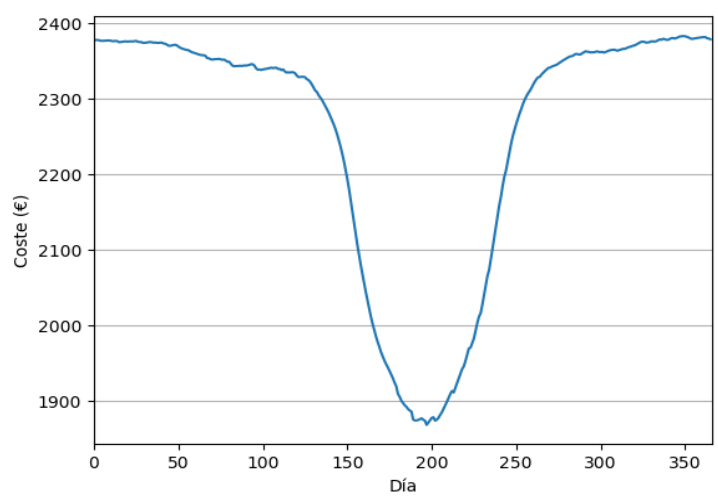

Figura 10: Coste en función del día de limpieza

\section{CONCLUSIONES}

En este trabajo se proponen formas de mejorar el mantenimiento de las instalaciones fotovoltaicas mediante la detección de fallos y la optimización de las fechas y frecuencias de limpieza.

Se ha explicado cómo calcular y utilizar varios índices $(P R$ y $R)$, para detectar fallos graves, así como otros de menos importancia acaecidos aguas abajo de la zona donde se dispone de datos de monitorización. Este método se ha aplicado a instalaciones reales, detectando diferentes averías y mostrando también el efecto de las reparaciones.

Por otra parte, se ha planteado una forma de optimizar las fechas de limpieza para cualquier instalación, que permite introducir diferentes perfiles de suciedad así como costes de la energía variables y diferentes probabilidades de lluvia. Por tanto, se puede adecuar el problema a cualquier instalación. La diferencia entre limpiar o no limpiar es en el caso más realista de $520.78 €$, por lo que dependerá del coste total de limpiar la instalación si resulta rentable hacer o no la limpieza.

\section{Agradecimientos}

Este trabajo ha sido parcialmente realizado bajo el proyecto "Dimensionado y uso óptimo de siste- 
mas de almacenamiento de energía en microrredes y redes de distribución inteligentes" (P1·1B201536), financiado por la Universitat Jaume I de Castellón.

\section{Referencias}

[1] International Renewable Energy Agency(IRENA), "Renewable energy statistics 2016," 2016.

[2] Fraunhofer ISE, "Photovoltaics Report," no. November, 2016.

[3] UNEF, "Informe Anual 2016," Unión Española Fotovoltaica, 2016.

[4] NREL, "Best Practices in Photovoltaic System Operations and Maintenance 2 nd Edition," no. December, 2016.

[5] B. Marion, J. Adelstein, H. Hadyen, B. Hammond, and T. Flecther, "Performance parameters for grid-connected PV systems," 31st IEEE Photovoltaics Specialists Conference and Exhibition, pp. 1601-1606, 2005.

[6] J. Zorrilla-Casanova, M. Piliougine, J. Carretero, P. Bernaola-Galvan, P. Carpena, L. Mora-López, and M. Sidrach-De-Cardona, "Losses produced by soiling in the incoming radiation to photovoltaic modules," Progress in Photovoltaics: Research and Applications, vol. 21, no. 4, pp. 790-796, 2013.

[7] M. García, L. Marroyo, E. Lorenzo, and M. Pérez, "Soiling and other optical losses in solar-tracking PV plants in navarra," Progress in Photovoltaics: Research and Applications, vol. 19, no. 2, pp. 211-217, 2011. 